\title{
DIFFRACTION-LIMITED IR SPECKLE MASKING OBSERVATIONS OF THE CENTRAL REGIONS OF SEYFERT
}

\author{
GALAXIES
}

\author{
M. WITTKOWSKI ${ }^{1,2}$, Y. BALEGA ${ }^{3}$, T. BECKERT ${ }^{2}$, W.J. DUSCHL ${ }^{2,1}$, \\ K.-H. HOFMANN ${ }^{1}$ AND G. WEIGELT ${ }^{1}$ \\ ${ }^{1}$ Max-Planck-Institut für Radioastronomie, Bonn, Germany \\ ${ }^{2}$ Institut für Theoretische Astrophysik, Heidelberg, Germany \\ AND \\ ${ }^{3}$ Special Astrophysical Observatory, Russia
}

We present speckle masking observations $([2],[4])$ of Seyfert galaxies with the Russian $6 \mathrm{~m}$ telescope. Diffraction-limited resolution of 76 mas in the $\mathrm{K}$-band was obtained for the first time. This resolution is similar to the resolution of recent MERLIN and VLA observations of galactic centers, thus allowing us to study the radio-IR spectrum of the same structures. Figure 1 shows the decreasing K-band visibility function of NGC 1068 and the contour plot of our reconstructed image ([5]). The results show that NGC 1068 is resolved with a FWHM diameter of 30 mas or 2 pc for an assumed Gaussian flux distribution. The image is elongated in northern direction, which is approximately the direction of the radio jet. In the right panel of figure 1 the observed flux values at 5,15 and $22 \mathrm{GHz}$ (from [3]) are plotted together with our K-band flux. The spectral index between $5 \mathrm{GHz}$ and the $\mathrm{K}$-band is approximately $1 / 3$. This spectrum can be explained by synchrotron emission of quasi-monoenergetic relativistic electrons (as for our Galactic Center by [1]). Assuming that the observed flux is mainly nuclear light (from, for example, scattering lobes above and below a torus, without absorption and re-emission) we use the same synchrotron model as has been used for the Galactic Center to explain our data. With this model, we find a source radius of $R \sim 10^{15} \mathrm{~cm}$, a magnetic field of $B \sim 11 \mathrm{G}$, a electron number density of $n_{e} \sim 1.110^{3} \mathrm{~cm}^{-3}$ and a mean electron energy of $\sim 2.7 \mathrm{GeV}$. The corresponding model spectrum is shown in the right panel of fig. 1 . The observed flux value at $2.2 \mu \mathrm{m}$ lies slightly above the model spectrum. This could be caused by flux contributions from additional components, for example, a central stellar cluster, an accretion disk or thermal radiation from a dusty torus. We have also observed the central regions of other galaxies. For example, for NGC 4151, we found a dominant 

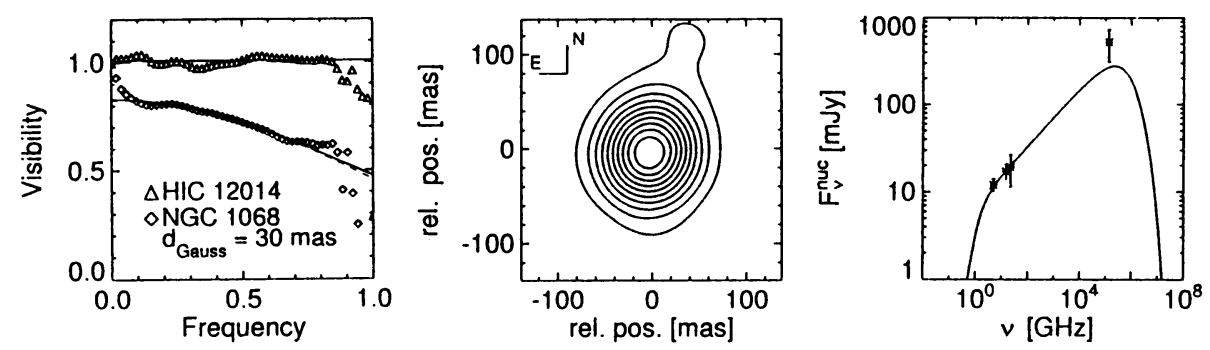

Figure 1. Left: Azimuthally averaged visibilities of NGC 1068 and the unresolved reference star. Middle: Contour plot of the reconstructed image. Right: A comparison of the model spectrum with the flux determinations at $5,15,22 \mathrm{GHz}$ and $2.2 \mu \mathrm{m}$.

central core, which is not resolved, and therefore has a diameter of $<20$ mas.

\section{References}

[1] Beckert T. et al., 1996, A\&A307, 450

[2] Lohmann A.W. et al., 1983, Appl. Opt. 22, 4028

[3] Muxlow T.W.B. et al., 1996, MNRAS 278, 854

[4] Weigelt G.P. 1977, Opt Comm. 21, 55

[5] Wittkowski M.. et al., 1997, submitted

M. DOPITA: You have not considered the possibility that the K-band object is simply the region of hot dust near its sublimation temperature. This should be visible, given that NGC 1068 is a hidden broad-line region (HBLR) Seyfert, and the sublimation radius for the object is $2-3 \mathrm{pc}$, similar to what you observe. Could you comment on the possibility that hot dust emission is the dominant emission process at $\mathrm{K}$ ?

M. WITTKOWSKI: Yes, this is another possibility. We make our hypothesis, because the spectral index is just very close to $1 / 3$ and a hot dust emission possibly peaks in the MIR and FIR and not already at $2.2 \mu \mathrm{m}$.

N. THATTE: NGC 1068 has a steeply rising spectrum in the K-band, which would need a spectral index $\alpha=4.9\left(S_{\nu} \sim \nu^{-\alpha}\right)$ to fit it. This is inconsistent with a synchrotron origin to the near-IR compact emission.

M. WITTKOWSKI: The K-band flux contain, of course, additional contributions, which could change the spectral index of the 30 mas source.

H. ZINNECKER: Have you also obtained H-band speckle images in order to determine the infrared colour of the 2 pc infrared core of NGC 1068 ? This would help to constrain its nature.

M. WITTKOWSKI: Yes, we have obtained H-band images. I agree, further observations with similar spatial resolution at other wavelengths are needed to constrain the models. 